\title{
Scleroderma - Lupus overlap syndrome: case report
}

\author{
Gabriela Ticu', Marius Ioan Balea ${ }^{2}$, Camelia Badea ${ }^{1,3}$, Adelina Silvana Gheorghe ${ }^{1,4}$, \\ Ruxandra Patrascu ${ }^{1,3}$ \\ ${ }^{1}$ Internal Medicine Department, Colentina Clinical Hospital, Bucharest, Romania \\ 2Pneumology Department, Colentina Clinical Hospital, Bucharest, Romania \\ 3"Carol Davila" University of Medicine and Pharmacy, Bucharest, Romania \\ 4"Prof. Dr. Alexandu Trestioreanu" Institute of Oncology, Bucharest, Romania
}

\begin{abstract}
The overlap syndromes are a diverse group of conditions that usually have characteristic features of at least two well-defined rheumatic diseases occurring together. We present the case of a 32-year-old patient who sequentially develops manifestations of systemic lupus erythematosus and systemic sclerosis. The first manifestation of the disease was the pleural effusion and, over time, the patient associated various other complications which influenced the diagnostic course. Digital ulcerations associated to scleroderma have evolved into ischemic necrosis lesions, requiring amputation. This case is a clinically suggestive example of the complexity of autoimmune diseases, of associations between pathologies and unexpected evolutions, requiring constant and long-term follow-up of the patients.
\end{abstract}

Keywords: systemic lupus erythematosus, serositis, systemic sclerosis, digital ulcers, overlap syndrome

\section{INTRODUCTION}

Clinicians are often challenged by the clinical issues when confronting with patients who either present an undifferentiated rheumatic disease or an overlap syndrome. In the medical literature, it is appreciated that approximately 25 percent of patients with rheumatic diseases cannot be definitely diagnosed with a single well-characterized systemic rheumatic disease $(1,2)$. Moreover, some of them remain undiagnosed for a long period of time, despite periodical reassessments and expectant management $(1,2)$. It happens due to the fact that this category of patients exhibits several patterns, manifesting multiple nonspecific serologic or clinical abnormalities, sometimes of more than one defined disorder. Some examples include rheumatoid arthritis - lupus (rhupus), scleroderma - polymyositis/dermatomyositis, scleroderma - lupus, scleroderma - rheumatoid arthritis, Sjögren's syndrome overlaps and others (1).

\section{CASE PRESENTATION}

We are presenting the case of a 32 years old woman, ex-smoker (10 pack-years, quit smoking 3 years ago), with class III obesity (body mass index $58.2 \mathrm{~kg} / \mathrm{m}^{2}$ ) and a history of abortion on demand one month prior to the admission at the hospital. She describes left posterior thoracic pain, night rigors, fever $\left(38.5^{\circ} \mathrm{C}\right)$, followed by decreased effort tolerance and dyspnea on minimal exertion, installed two weeks before presentation. The patient also complains of morning joint stiffness, especially in the wrists, which had intensified in the last month.

From the pathological personal history, it is worth mentioning that the patient followed 6 months of tuberculostatic treatment, 9 years ago, for positive Mantoux tuberculin skin test, but without suggestive chest radiography or confirmation of tuberculosis through sputum smear microscopy.

Physical examination on admission to the hospital revealed that the patient was afebrile, tachypneic 
with a respiratory rate of 24 breaths per minute and tachycardic with a ventricular rate of 104 rhythmic beats per minute (regular heart rhythm). The vesicular murmur was abolished with dullness to percussion over left lung base and decreased breath sounds bilaterally. A bilateral hand edema could also be observed. The blood pressure was normal, without pulmonary crackles, cardiac murmurs or extra sounds and no evidence of arthritis or rashes.

Since the onset of symptoms, prior to the admission in the hospital, the patient presented to the general practitioner, who recommended chest radiography, computed tomography (CT) scan of the thorax and pleural ultrasound. At these investigations, a small pleural effusion was discovered in the left lung, along with atelectasis aspect or pulmonary parenchyma condensation (Fig. 1). No direct or indirect signs of pulmonary embolism have been detected.

The episode was interpreted as the an intercurrent respiratory infection, with secondary pleural reaction and the patient received broad-spectrum empiric antibiotic therapy: 6 days of levofloxacin, followed by 3 days of ciprofloxacin and 5 days of drug combination (gentamicin, amoxicillin/clavulanic acid and metronidazole). Under this treatment, she continued to develop fever $\left(38.2-38.5^{\circ} \mathrm{C}\right)$.

We identified an important inflammatory process, based on the elevation of serum biomarkers: C-reactive protein $(146.55 \mathrm{mg} / \mathrm{L})$, erythrocyte sedimentation rate $(61 \mathrm{~mm} / 1 \mathrm{~h})$ and fibrinogen $(915 \mathrm{mg} /$ $\mathrm{dL})$. The complete blood count showed discrete anemia, thrombocytosis, without leukocytosis or leukopenia. Serum creatinine and microscopic urinalysis were normal.

We also performed chest radiography after admission, which revealed the same aspect of pleural effusion in small amounts, located in the left costo- diaphragmatic recess. The rest of the investigations (electrocardiogram, echocardiogram, abdominal and pelvic ultrasound, lower extremity veins Doppler ultrasound) did not show any relevant particularities.

The patient underwent thoracentesis of the left pleural effusion. The fluid obtained had the characteristics of an exudate, with 2.400 cells $/ \mathrm{mm}^{3}, 46 \%$ neutrophils and 27\% lymphocytes, but with negative bacterial cultures. Examination by Ziehl-Neelsen staining did not reveal acid-alcohol-resistant bacilli, the level of adenosine deaminase in the effusion liquid was normal and Mycobacterium tuberculosis DNA was not detected by polymerase chain reaction. Also, the histopathological examination of the pleural biopsy was normal.

Despite having received antibiotic (ceftriaxone), anti-inflammatory and antialgic (celecoxib) treatment, subfebrility persisted, only with a minimal improvement of the thoracic pain.

After 10 days, the patient was admitted again in the hospital for paroxysmal nocturnal dyspnea, persistence of the thoracic pain and dyspnea on minimal exertion. Clinical examination and several investigations (thoracic ultrasound, echocardiography and CT scan) led to the discovery of pericardial and bilateral pleural effusions. During thoracic ultrasound, an important right diaphragmatic elevation with low mobility of the diaphragm was also noticed.

The serological tests revealed positive anti-nuclear antibodies, C3 hypocomplementemia, presence of lupus anticoagulant and high-titer anti-cardiolipin IgM antibodies (antiphospholipid antibody positivity). Anti-double stranded DNA (dsDNA) antibodies, anti-Ro antibodies, anti-beta2-glycoprotein I IgG antibodies, anti-cardiolipin IgG antibodies were negative, as well as the rheumatoid factor and direct Coombs test.

These three immunological criteria, added to the forth clinical criterion (serositis, consisting in pleuri-

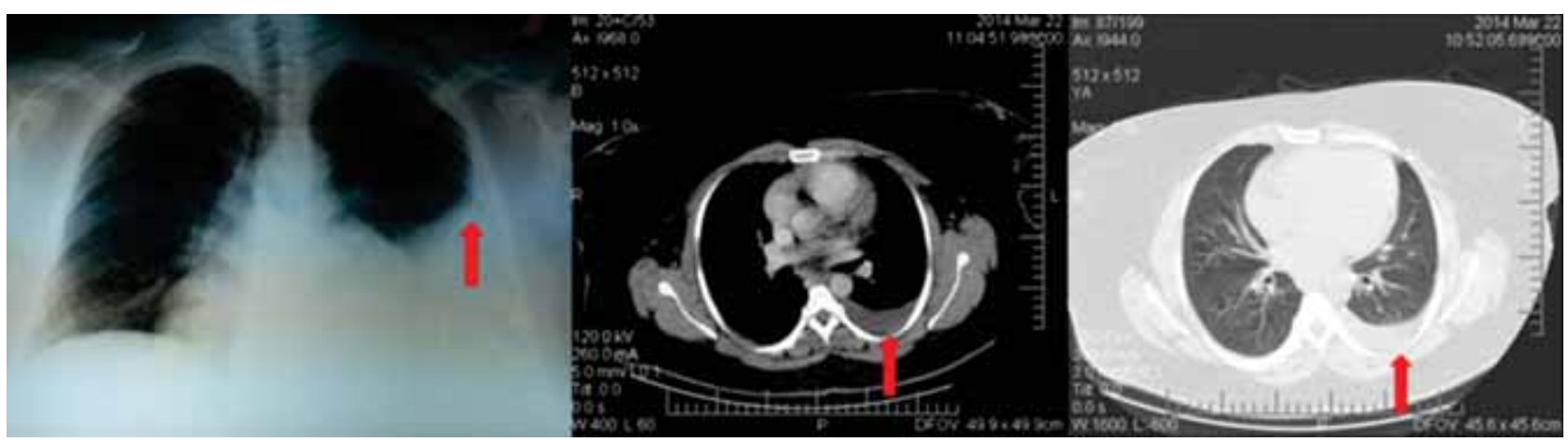

FIGURE 1. Chest radiography and computed tomography scan of the thorax showing small pleural effusion in the left lung (with a maximum of 2 centimeters in transversal diameter at the ultrasound examination) 
sy and pericarditis), led to the classification and then diagnosis of systemic lupus erythematosus (SLE) with minimal articular involvement (based on the aforementioned morning joint stiffness) - according to SLICC (Systemic Lupus International Collaborating Clinics) classification criteria 2012. Due to the presence of progressive dyspnea, accompanied by pleuritic chest pain, diaphragmatic elevation and lung volume reduction, we suspected a rare complication associated with lupus, the shrinking lung syndrome, but this has not been confirmed.

Under treatment with systemic corticosteroids (Dexamethasone, followed by Methyl-prednisolone) and anti-malarial drug (Hydroxychloroquine), the patient had a favorable evolution, both clinically and biologically.

Over the following two months, the patient did not experience fever, dyspnea or thoracic pain, so we advised her to continue the treatment with corticosteroid and hydroxychloroquine as well as to lose weight.

The patient was admitted to the hospital again, after two and a half years when she presented acrocyanosis with trophic changes and ulcerations in the distal phalanges of the hands, persistent over the previous months. Meanwhile, she underwent gastric sleeve surgery and started smoking again.

Physical examination on this admission to the hospital found a normoponderal patient (body mass index $23.44 \mathrm{~kg} / \mathrm{m}^{2}$ ) with puffy fingers, sclerodactyly of the fingers, digital tip ulcers, fingertip pitting scars, severe acrocyanosis of the hands and telangiectasia.

Immunological markers showed low C3, normal levels of anti-centromere and anti-La antibodies, absence of lupus anticoagulant and absence of cryoglobulins. The other serological tests had resulted to be the same as the ones from the previous admission.

The electrocardiogram revealed a minor form of right bundle branch block, while a slight interventricular septum hypokinesis had been seen on heart ultrasound. There were no radiological changes on hand radiography, but nailfold capilloscopy brought to light a "late" scleroderma pattern.

Changes in pulmonary imaging were suggestive of fibrosis (diffuse "ground-glass" opacification, pulmonary alveolar infiltrates and bronchiectasis). In addition, alveolar-capillary membrane diffusing capacity was normal. Pelvic ultrasound by transabdominal evaluation showed abnormal uterus and right ovarian masses, therefore we recommended a pelvis magnetic resonance imaging (MRI) scan.
The switch in the patient's dominant signs and symptoms guided us to discover a second autoimmune illness: systemic sclerosis (SSc) or scleroderma. Meeting the classification criteria for two separate well-characterized rheumatic diseases, SLE and SSc, we came to the final diagnosis of overlap syndrome between the two diseases.

The patient received treatment with corticosteroid, anti-malaric, sulodexide and pentoxifylline, as well as topical dermatological preparations for the ischemic lesions. She was advised to avoid cold exposure and to quit smoking. No further actions were taken for the next 5 months, as the patient did not present to the hospital for immunosuppressive therapy and association of anticoagulation. During this time, she underwent gynecological assessments in the private healthcare system. The cervical biopsy showed the presence of an intraepithelial squamous lesion, positive for Human papillomavirus DNA (high-risk genotype).

At the following admission to the hospital, the physical examination showed that the lesions of ischemic necrosis had advanced. Using an endovaginal ultrasound, pelvic intraperitoneal fluid and ovarian inhomogeneous tumoral mass were detected, without a concrete diagnosis of the gynecological findings.

The previously suggested line of treatment was applied: we added acenocoumarol for the ischemic necrosis lesions and started the intravenous cyclophosphamide pulse therapy, at a dose calculated according to the weight and body surface.

By the time of administrating the second cyclophosphamide pulse therapy, the patient presented with extensive digital necrosis (first, second, third fingers of the left hand and second, third, fourth of the right one). We considered that the clinical evolution of peripheral ischemic lesions has dramatically worsened, which is why we suggested it was appropriate to amputate the necrotic lesions, discontinue the cyclophosphamide pulse therapy and revalue the patient's status after surgery. She was then admitted in the plastic surgery department for debridement therapy of the wounds; systemic vasodilator treatment and amputation of the distal phalanges of fingers II and III (right hand) were necessary.

After 2 months, the patient presented for continuation of cyclophosphamide pulse therapy, when coupled repetitive monomorphic supraventricular and ventricular extrasystoles were observed for the first time on the electrocardiogram. 


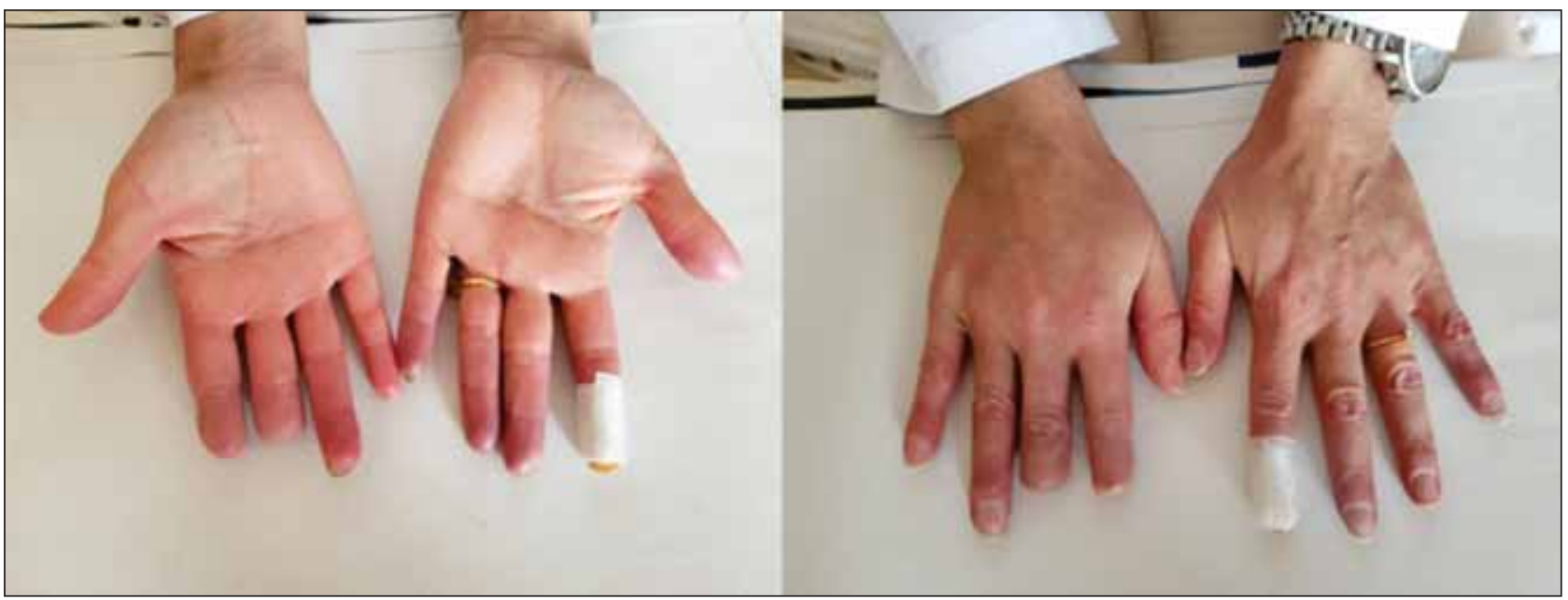

FIGURE 2. Raynaud's phenomenon (acrocyanosis), 3 months after the amputation of distal phalanges of fingers II and III from the right hand

Another month later, the evolution of the patient seemed to be favorable, without dyspnea, arthritis, arthralgia, myalgia, but with severe and persistent acrocyanosis in the hands and feet, along with acroparesthesia at cold exposure (Fig. 2).

The patient developed atrial flutter with variable block, heart rate ranging from 60 to 90 beats per minute and value of the systolic blood pressure between 80 and $90 \mathrm{mmHg}$. On repeated electrocardiograms, atrial arrhythmia remained present and therefore we associated a non-dihydropyridine calcium channel blocker to the treatment strategy. Beta-blocker was not added, due to the severe peripheral manifestations.

The following four cyclophosphamide pulse therapies took place without any adverse reactions or supplementary findings, considering that the clinical progression was favorable. At the last admission to the hospital, there was no evidence of atrial flutter on the electrocardiogram, but several coupled supraventricular extrasystoles were detected during the Holter monitoring.

During these admissions to the hospital, there were some constant findings: negative lupus anticoagulant, no inflammatory biological syndrome and low C3 with normal C4 levels. The patient did not develop other complications of SSc or SLE, except for Raynaud's phenomenon. We have been monitoring the possible development of pulmonary arterial hypertension, but there have not been signs of this complication. After the last cyclophosphamide pulse therapy, the SLEDAI severity score (Systemic Lupus Erythematosus Disease Activity Index) was 2, which meant that the disease was inactive. Moreo- ver, the patient also underwent periodical ophthalmological monitoring and she did not manifest any changes.

After two months since the sixth cyclophosphamide pulse therapy, the patient was admitted again to the hospital for the seventh immunosuppressive drug administration. On the electrocardiogram, sinus rhythm was maintained, with coupled supraventricular extrasystoles and a heart rate of 70-80 beats per minute.

We initiated dual endothelin receptor antagonist (Bosentan) therapy for preventing further digital ulcers caused by SSc, and the patient would be reevaluated in one month. On initiation, we performed an assessment of the digital tip ulcers and fingertip pitting scars of the hands: ulcers were not present, only scars without calcification in right finger IV $(4 \mathrm{~mm})$, right finger V (3 mm), left finger I (5 mm), left finger II (11 mm), left finger III (4 mm), left finger IV (3 $\mathrm{mm})$, left finger $\mathrm{V}(5 \mathrm{~mm})$. We evaluated the HAQ-DI (Health Assessment Questionnaire Disability Index) and VAS (Visual Analogue Scale) regarding the interference of Raynaud's phenomenon and digital ulcers with daily activities. HAQ-DI score was 0 , while VAS for Raynaud's phenomenon was $10 \mathrm{~mm}$ and for digital ulcers $30 \mathrm{~mm}$. Upon monitoring the efficacy and toxicity response to the drug, we will decide whether the patient should continue the treatment.

We questioned the problem of differential diagnosis with mixed connective tissue disease (MCTD), for which some of the Alarcon-Segovia and Villareal Diagnostic Clinical Criteria are met (edema of the hands, Raynaud's syndrome and acrosclerosis). 
Consequently, we aimed to detect the presence of anti-U1 ribonucleoprotein (RNP) antibodies (the serologic criteria for MCTD), which proved to be negative.

\section{DISCUSSIONS}

Up to a third of patients with SLE might develop another autoimmune disease, which can have an important impact on the development of damage and mortality. (3) For example, it has been shown that patients with SLE and other autoimmune disease have worse damage scores at 5 years after the lupus diagnosis, when compared to patients with SLE only (3).

Despite the fact that ample epidemiologic studies on the incidence and prevalence of overlap syndromes have not been done yet, there are some available data. One retrospective study which analyzed 1,321 patients, demonstrated that an overlapping second autoimmune illness (rheumatic or non-rheumatic) occurs in $30-52 \%$ of patients who already have a diagnosis of SLE, rheumatoid arthritis, Sjögren's syndrome or antiphospholipid syndrome. (4) In addition, the study shows that there is a difference in terms of demographic aspects and treatment algorithms between patients with "pure" disease and those with second autoimmune illness (4).

In rheumatology, it is hard to establish criteria characteristic for each separate disease, due to the lack of "gold standards". Different criteria (of classification/diagnosis, response and remission) intend to guide clinicians in establishing diagnoses and choosing the right treatment, but we sometimes have to tolerate the uncertainty and avoid diagnostic labels if they are not clearly supported by established criteria (2).

From the beginning, the case was challenging in terms of differentiating between the infectious and purely inflammatory process of the pleural effusion. After ruling out the most frequent causes of exudative effusions (pneumonia, cancer, tuberculosis and pulmonary embolism), an autoimmune disease was taken into consideration, as serositis is one of both ACR (American College of Rheumatology) and SLICC classification criteria for SLE (5). This manifestation is not one of the most common ones in SLE, but up to half of the patients (12-45\%) present with either pleurisy or pericarditis (6). Pleuritis as the initial presentation to the hospital was found in more than one third of the patients included in a ret- rospective analysis of pleural effusions, out of which $52 \%$ were associated to SLE, $9 \%$ to tuberculosis and $7 \%$ to parapneumonic processes (7). A cross-sectional and prospective study including 2,390 SLE patients showed that fever, Raynaud's syndrome and anti-dsDNA antibodies were predictive factors of both pleurisy and pericarditis in SLE (6).

Besides pleuritis, there are also other pleuropulmonary manifestations which occur in $60-80 \%$ of patients with SLE. Out of these, shrinking lung syndrome has an estimated prevalence between $0.5 \%$ and $1.1 \%$ in SLE population, more common in women (17:1 female:male ratio) (8). It is reported to be much more frequent in advanced stages of the disease, but there are also rare cases of being the presenting manifestation of SLE (8). There is literature evidence of shrinking lung syndrome in patients with SLE-SSc overlap syndrome and therefore, this rare condition should be taken into consideration (9).

Rhythm and conduction disorders, along with sudden cardiac death are important manifestations of the cardiac involvement in autoimmune rheumatic diseases. The main pathophysiological mechanisms which leads to arrhythmias are the autoimmune process itself, atherosclerotic complications and even side-effects of the treatment (10). Sinus tachycardia, atrial fibrillation and atrial ectopic beats are the major cardiac arrhythmias described in SLE patients, along with sick sinus syndrome and long QT syndrome $(10,11)$. Our patient presented with sinus tachycardia at the first admission to the hospital. In some cases, sinus tachycardia may be the single manifestation of cardiac involvement (11). However, in our case report, it was followed by a minor form of right bundle branch block and paroxysmal atrial fibrillation. The most common heart rhythm disorders in SSc are monomorphic, single premature ventricular contractions, but also transient atrial fibrillation, atrial flutter or paroxysmal supraventricular tachycardia have been described in $20-30 \%$ of these patients (11). Moreover, 25-75\% of patients with SSc develop conduction disturbances (bundle and fascicular blocks), due to fibrosis of the sinoatrial node (11).

There are studies that demonstrate the influence of diet on SLE patients, based on the hypothesis of an existing molecular link between diet, gut commensal germ populationm and immunity (12). It has been shown that dietary changes can significantly affect the composition of gut microbiome and virome, which possibly contributes to SLE pathogene- 
sis (12). The patient from our case report has undergone drastic changes in her diet, especially after the gastric sleeve surgery. Caloric restriction was appreciated to have positive effects and prevent the progression of SLE, as well as other SLE associated autoimmune diseases (12).

\section{CONCLUSIONS}

The intricate diagnostic course and the dynamic association of new elements have made this case to be a challenging one. In addition to this, the complications that occurred are complex, notably the unfavorable evolution of digital ulcers to amputation in a

\section{REFERENCES}

1. Panush R.S., Kramer N., Rosenstein E.D. Undifferentiated systemic rheumatic (connective tissue) diseases and overlap syndromes. UpToDate. 2018

2. Kelly A., Panush R.S. Diagnostic uncertainty and epistemologic humility. Clin Rheumatol. 2017;36(6):1211-1214.

3. Chambers S.A., Charman S.C., Rahman A., Isenberg D.A. Development of additional autoimmune diseases in a multiethnic cohort of patients with systemic lupus erythematosus with reference to damage and mortality. Ann Rheum Dis. 2007;66(9):1173-1177.

4. Lockshin M.D., Levine A.B., Erkan D. Patients with overlap autoimmune disease differ from those with "pure" disease. Lupus Sci Med. 2015;2(1):e000084.

5. Porcel J.M., Light R.W. Diagnostic approach to pleural effusion in adults. Am Fam Physician. 2006;73(7):1211-1220.

6. Ryu S., Fu W., Petri M.A. Associates and predictors of pleurisy or pericarditis in SLE. Lupus Sci Med. 2017;4(1):e000221.

7. Palavutitotai N., Buppajarntham T., Katchamart W. Etiologies and outcomes of pleural effusions in patients with systemic lupus erythematosus. J Clin Rheumatol. 2014;20(8):418-421. woman of young age, the development of paroxysmal atrial flutter under the anticoagulant treatment received for peripheral ischemic necrosis and the association of gynecological pathology, still of undetermined etiology. The physical transformation of the patient is spectacular and manifests a strong will in fighting with the disease, with a weight loss from $149 \mathrm{~kg}$ to $56 \mathrm{~kg}$ at the present time.

This case report emphasizes on the co-existence of multiple autoimmune diseases in individual patients, a fact that clinicians should be aware of, in order constantly to adapt the frequency and specifics of the patient follow-up.

Conflict of interest: none declared Financial support: none declared

8. Borrell H., Narváez J., Alegre J.J. et al. Shrinking lung syndrome in systemic lupus erythematosus: A case series and review of the literature. Medicine. 2016;95(33):e4626.

9. Guleria V.S., Singh P.K., Saxena P., Subramanian S. Shrinking lung syndrome in systemic lupus erythematosus-scleroderma overlap. Lung India. 2014;31(4):407-409.

10. Teixeira R.A., Borba E.F., Bonfá E., Martinelli Filho M. Arrhythmias in systemic lupus erythematosus. Rev Bras Reumatol. $2010 ; 50(1): 81-89$

11. Seferović P.M., Ristić A.D., Maksimović R. et al. Cardiac arrhythmias and conduction disturbances in autoimmune rheumatic diseases. Rheumatology (Oxford). 2006;45(Suppl4):iv39-42.

12. Vieira S.M., Pagovich O.E., Kriegel M.A. Diet, Microbiota and Autoimmune Diseases. Lupus. 2014;23(6):518-526. 